\title{
Die Kultur von digitalen Nomaden im Kontext der Ontologie der Netzwerkgesellschaft und Kultursemiotik
}

\author{
Irina Petrovna Kuzheleva-Sagan, Tomsk State University
}

\begin{abstract}
Digital nomadism is a new way of life that became possible only at the period of network society owing to the appearance of wireless communication technology. It has two main characteristics: permanent mobility on global or local levels and connection (or being "plugged") to networks. Digital nomads are people who due to using Internet and mobile technologies are not tied to a definite place and who conduct their professional life, study or do creative work in a nomadic manner (Makimoto und Manners 1997). The ontology of the modern society, its technologization, informatisation, computerization, use of the digital technologies and networking encourage the development of digital nomadic culture and helps to promote it as a universal culture of the network society. This fast development causes different problems. One of these problems is that the digital nomadic culture is far ahead of the research and description of its philosophical and theoretical grounds that would give an opportunity for a relevant analysis of its specific character and functioning mechanisms. The purpose of this article is to represent the traits of the digital nomadic culture. The general description of their culture in context of network society is based on the traditions of culture semiotics as understood by J. Lotman and R. Posner. The author of this article assumes that this approach can help not only to define the identity, borders and factors of the existence of digital nomadic culture, but provide an account of it as a comprehensive and globalized phenomenon as well.
\end{abstract}

Zusammenfassung. Das digitale Nomadentum ist eine neue Lebensweise, die durch das Zusammentreffen von Netzwerkgesellschaften mit drahtloser Kommunikation ermöglicht wurde. Diese Lebensweise weist zwei Hauptmerkmale auf: die ständige globale oder lokale Mobilität sowie permanente Konnektivität oder Erreichbarkeit. Digitale Nomaden sind somit Menschen, die in ihrem beruflichen Altag, im Studium oder bei ihren Freizeitbeschäftigungen dank Internettechnologien und Mobilfunknetz ortsunabhängig sind (Makimoto und Manners 1997). Die Technologisierung und Informatisierung der modernen Gesellschaft, der Einfluss von Computern, digitalen Technologien und Netzwerken tragen zur Entstehung der Kultur von digitalen Nomaden bei. Allmählich etab- 
liert sie sich und wird zur universellen Kultur der Netzwerkgesellschaft. Die wissenschaftliche Beschreibung dieser Kultur erweist dich noch als lückenhaft. Die Kulturpraktiken von digitalen Nomaden überholen die Erarbeitung ihrer theoretischen und philosophischen Grundlagen. Dadurch wird die adäquate wissenschaftliche Erfassung dieser Praktiken und Mechanismen verhindert. Aus diesem Grund wird in diesem Artikel der Versuch unternommen, die allgemeinen Eigenschaften der Kultur von digitalen Nomaden zu bestimmen. Die Darstellung dieser Kultur im Rahmen der Netzwerkgesellschaft basiert auf der Auffassung der Kultursemiotik im Sinne von J. Lotman und R. Posner. Dabei wird vorausgesetzt, dass die oben genannte Herangehensweise bei der Bestimmung der Identität, Grenzen und Bedingungen der Kultur von digitalen Nomaden hilft. Außerdem dient sie der Darstellung dieser Kultur in ihrer Gesamtheit als eine sich ausbreitende Erscheinung.

\section{Einführung}

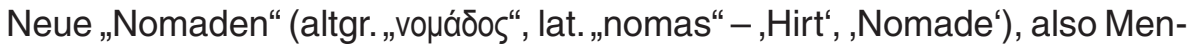
schen, die Informationen mit Hilfe von digitalen Medien sammeln, wurden zum ersten Mal vom kanadischen Medientheoretiker McLuhan bereits in den 60er Jahren des 20. Jahrhunderts erwähnt. Etwas später wird die Bezeichnung „Nomade“ zu einem Schlüsselbegriff der Nomadologie - der metaphorischen postmodernistischen Konzeption „Neuer Tribalismus“ von G. Deleuze und F. Guattari. Das war ein Ergebnis ihrer philosophischen Auseinandersetzung mit der Opposition „Macht des Staates“ versus „Freiheit des einzelnen Individuums“. Auf der Suche nach einem Gegengewicht zur Macht des Staates sind Deleuze und Guattari auf die NomadismusIdee gekommen. Wie aus der Geschichte bekannt, waren Nomaden in der Lage, die stärksten Imperien zu zerstören. Laut der Hypothese der französischen Philosophen hat die Kultur des Hirtenvolkes eine besondere Eigenschaft, und zwar die angeborene Fähigkeit, eine Kriegsmaschine zu schaffen, die gegen den Staatsapparat antritt.

Die Anmaßung des Staates, das verinnerlichte Bild einer Weltordnung zu sein und den Menschen zu verwurzeln. Aber die Beziehung einer Kriegsmaschine zu einem Außen ist kein anderes „Modell“, es ist ein Gefüge, das das Denken selber nomadisch macht und das Buch zu einem Teil aller beweglichen Maschinen, zu einem Strang für ein Rhizom (Deleuze und Guattari 2005: 40).

Mit anderen Worten kann jeder freie Gedanke zur Kriegsmaschine oder zum Hirtenvolk werden und dem dominierenden Kulturcode Widerstand leisten. Die Metapher „nomadisches Denken“ wurde von den Philosophen als Antithese von einem Denken, das einen „Standpunkt" hat (Sedentarismus), eingeführt. Letzteres ist für jede Art von festgelegten Lebensweisen charakteristisch und spiegelt sich in der Idee des Staates wider. Der Nomadismus führt zur Deterritorialisierung der Gesellschaft und zur allmählichen 
Auflösung ihres Zentrums. Die „Nomadologie“ von Deleuze und Guattari wurde zu einer der bedeutendsten postmodernistischen Konzeptionen und der Begriff „Nomade“ wurde zu einem sehr oft benutzten Konzept im 20. Jahrhundert (vgl. Meyrowitz 1985; Bauman 1993; Braidotti 1994). Deshalb konnte der Medienhistoriker und Sozialtheoretiker J. D. Peters mit Recht behaupten, dass „der Nomade eindeutig der Held des postmodernistischen Denkens" ist (Peters 1999: 33).

Der französische Soziologe und Verfechter des Globalismus Jacques Attali beschäftigt sich in seinem Buch Millennium: Winners and Losers in the coming world order (Attali 1991) mit den sozialen, politischen, wirtschaftlichen und zum Teil auch anthropologischen Seiten des neuen Nomadentums. Er behauptet, dass sich zu Beginn des neuen Jahrtausends die digitale nomadische Form der Wirtschaft als hyperindustrielle Gesellschaft etabliert, und entwickelt das Konzept der Nomadenobjekte. Das sind die günstigen und kompakten (mobilen) Endgeräte, die über viele Funktionen verfügen und essentiell für die mobilen Mitglieder der globalen technikfreundlichen Gesellschaft sind. Attali schreibt über die weitere Entwicklung von Nomadenobjekten und spekuliert, dass es einen Kulturwandel geben werde. Im Laufe dieser Mutation und durch den Austausch der alten nicht funktionierenden Organe durch neue künstliche wird der Mensch eines Tages auch zu einem Nomadenobjekt (vgl. Attali 1991: 11).

Eine erneute Welle des Interesses an diesem Thema gab es Ende der 1990er Jahre. Sie wurde durch die Veröffentlichung des Buches Digital Nomad von T. Makimoto und D. Manners ausgelöst. Nach den Autoren des Buches gilt: Digitale Nomaden sind Menschen, die in ihrem beruflichen Alltag, im Studium oder bei ihren Freizeitbeschäftigungen dank Internettechnologien und Mobilfunknetz ortsunabhängig sind (Makimoto und Manners, 1997). Einige Zeit später schrieb der Kunsthistoriker W. J. Mitchell:

By the early 2000s, many workers (and their employers) had discovered that they just needed a cellphone and a laptop to operate effectively at their nominal workplaces, on commuter trains, in airplane seats and airport lounges, in hotel rooms, at the sites of clients and collaborators, at home, and on vacation. Anyplace was now a potential workplace. And this condition would only intensify as the technology of nomadics developed and proliferated (Mitchell 2003: 153).

Der metaphorische Ausdruck „digitale Nomaden“ („digital nomads“, DN) kommt immer öfter in privaten und Firmenblogs, aber auch in den Printmedien vor (The Times 1998, Economist 2008, Forbes 2014). Überdies wird er öfter in interdisziplinären Kontexten als Fachterminus von Soziologen (vgl. Urry 2000), Sozialpsychologen (vgl. Rosseel 2000), Sprachwissenschaftlern (vgl. Baron 2001), Wirtschaftswissenschaftlern (vgl. Kakihara und Sorensen 2001), Medientheoretikern (vgl. Mitchell 2003) und Architekten (vgl. Celento 2008) verwendet. Häufig wurde die Lebensweise der digitalen Nomaden von den Herstellern mobiler Endgeräte und Technologien popularisiert und romantisiert. In Wirklichkeit werden digitale Nomaden 
dagegen zumindest in manchen Fällen als gesellschaftliche Außenseiter, Exzentriker und Freaks wahrgenommen. Inzwischen gibt es immer mehr davon. Zuerst waren es Einwohner der Millionenstädte, wo das WLAN zum Alltag gehört. Die Entwicklung der Satellitenkommunikation ging weiter und nun trifft man digitale Nomaden sowohl auf dem Dorf als auch in der Wüste oder Tundra. Wer führt ein nomadisches Leben auf globaler oder lokaler Ebene innerhalb einer Stadt? Das sind nicht nur Einzelgänger, die als Freelancer arbeiten und ein sehr niedriges Einkommen haben. Für diesen Lebensstil entscheiden sich auch finanziell wohlhabende Menschen, zum Beispiel Eigentümer von Firmen, Führungskräfte von Unternehmen ohne Büro, gut bezahlte Experten und Berater, Schriftsteller und Autoren von Bestsellern usw. Vom sozialen Status unabhängig haben alle diese Personen eins gemeinsam. Sie sind ständig unterwegs und sind stets per Mobilfunk oder via Internet erreichbar. Allerdings ist zu betonen, dass der Lebensstil der „Digitalen Nomaden“ mittlerweile auch von anderen Berufsgruppen, etwa von festangestellten Lehrern, Akademikern oder normalen Angestellten übernommen wird.

Der digitale Nomadismus als Erscheinung existiert ausschließlich in der Informations-, Kommunikations- und Netzwerkgesellschaft (Castells 1996). Die ständige Mobilität und Erreichbarkeit von digitalen Nomaden macht den größten Teil ihrer Identität aus. Diese Eigenschaften lassen sich von den Nomaden auf ihre Kultur übertragen. Sie entwickelt sich sehr schnell und ihre schnelle Verbreitung findet nicht nur horizontal innerhalb derselben sozialen Schichten statt, sondern umfasst vertikal verschiedene Strata. Als Ergebnis „sind wir die Zeugen eines Rachefeldzugs des nomadischen Prinzips gegen die Prinzipien der Territorialität und Seßhaftigkeit“" (Bauman 2000: 20f.). Seitdem die soziokulturelle Erscheinung existiert und immer mehr an Beliebtheit gewinnt, wurde das neue Nomadentum relativ selten in wissenschaftlichen Auseinandersetzungen und der Forschung thematisiert (vgl. Johannessen 2000; Rosseel 2000; Wood 2005; de Lange 2009). In der Regel stehen im Mittelpunkt der Forschungsinteressen einzelne Aspekte der Kultur von digitalen Nomaden, beispielsweise ihre Sprache (Baron 2001), Nomadenobjekte (Braidotti 1994; Fortunati 2002), Mobilität (Kakihara und Sorensen 2001) usw. Demzufolge steht eine umfassende ganzheitliche wissenschaftliche Beschreibung der Kultur von digitalen Nomaden trotz ihrer rasanten Verbreitung noch aus. Die Ursache dafür kann in den fehlenden oder noch nicht genügend erarbeiteten philosophischen und theoretischen Grundlagen vermutet werden, die eine gründliche Analyse der Spezifik und der Mechanismen dieser Kultur ermöglichen würden.

Im Weiteren wird der Versuch unternommen, die Besonderheiten der Netzwerkgesellschaft als Kontext für die Entstehung und das Funktionieren der Kultur von digitalen Nomaden zu erfassen. Überdies werden die bedeutendsten Aspekte der Kultursemiotik im Sinne von J. Lotman und R. Posner zusammengefasst. Sie dienen als die methodologische Basis für die weitere Analyse der Nomadenkultur, die als ein evolutionäres Ganzes betrachtet wird. 


\section{Ontologie der Netzwerkgesellschaft als Ontologie des digitalen Nomadismus}

Um das Wesen des digitalen Nomadentums und seiner Kultur zu verstehen, sollte man sich mit der Ontologie, also mit der Natur der Netzwerk-, Informations- und Kommunikationsgesellschaft näher beschäftigen. In Bezug auf die Ontologie dieser Gesellschaft, ihre Bestandteile, Vorgänge und Trends, zu denen auch der digitale Nomadismus zählt, existieren mindestens zwei wesentliche Positionen. Kurz zusammengefasst kann man sie wie folgt darstellen (siehe auch Kuzheleva-Sagan 2014).

\subsection{Utopische Position}

Die ersten Vorstellungen oder viel mehr Antizipationen einer neuen Gesellschaft gab es lange vor ihrer Entstehung. Ohne die Komplexität der Netzwerkgesellschaft und ihre potentiellen Probleme abzustreiten, vertraten optimistische Futurologen und Technokraten - z.B. D. Bell (1973), Z. Brzezinski (1970), J. K. Galbraith (2007), M. McLuhan (1994), J. Naisbitt (1982), A. Toffler (1980) - die Meinung, dass diese Probleme mit Hilfe der neuesten elektronischen und Computertechnologien gelöst werden. Die bessere Zukunft verband man mit der nicht hierarchischen Struktur der Netzwerkgesellschaft und der Blüte der Wirtschaft des neuen Typs, die wissens- und informationsbasiert sowie serviceorientiert ist, und außerdem mit dem Ende der Bürokratie und dem Triumph der Demokratie. Die Schlüsselideen von optimistischen Futurologen und Technokraten bezüglich des Lebens- und Arbeitsraums lassen sich wie folgende Metaphern zusammenfassen: „Global village“ (McLuhan 1994), „global metropolis“ (Kahn und Bruce-Briggs 1972), „global city“ (Sassen 2001), „electronic cottage“ (Toffler 1980). Alle diese Ausdrücke beschreiben die ty $\mathrm{p}$ is chen Orte der Arbeit und des Lebens und stehen in keinem Widerspruch zur Existenz des Menschen. Verändert haben sich nur ihre Größe und technische Ausstattung. Aber genau diese Faktoren haben zur Folge, dass Menschen über neue faszinierende Möglichkeiten verfügen. Diese Vorstellung des Weltbildes der Zukunft dominierte in der Sozialphilosophie, Soziologie und Futurologie im letzten Drittel des 20. Jahrhunderts.

\subsection{Dystopische Position}

Vertreten wird diese Position von technophoben Futurologen, Existenzphilosophen, Theoretikern der Frankfurter Schule, Poststrukturalisten und Postmodernisten sowie von einigen Soziologen, Wirtschafts- und Medienwissenschaftlern: z.B. A. Huxley (2005), G. Orwell (2003), K. Jaspers (1953), H. Marcuse (1991), J. Baudrillard (1981), P. Virilio (2010), S. Garfinkel (2000), J. Attali (1991), A. Bard und J. Soderqvist (2002), W. J. Mitchell (2003) sowie 
F. Guattari und G. Deleuze. Bei aller Verschiedenheit der wissenschaftlichen Gebiete sind sie sich darin einig, dass sie nicht versuchen, in ihren ontologischen Beschreibungen die postindustrielle Gesellschaft in rosigen Farben auszumalen. Außerdem betonen sie die ambivalente Natur der Technologien, die außer Kontrolle geraten und Menschen offen oder verborgen steuern können. Darüber hinaus machen sich die Vertreter dieser Ansicht keine Illusionen bezüglich der Transparenz und der fehlenden Hierarchien in der Netzwerkgesellschaft.

Die Ontologie der Netzwerkgesellschaft kann in diesem Fall als digitale, informations- und kommunikationsbasierte, sich selbst organisierende Struktur charakterisiert werden. Sie beruht auf den Prinzipien der Ordnung und des Chaos wie organisierte Simulakren. Eine der wichtigsten Eigenschaften der Netzwerkgesellschaft ist die Hybridität des Raums, d. h. es gibt keine klaren Grenzen zwischen real und virtuell. Ihre Struktur lässt sich als hierarchische, netzartige, rhizomatische, neuronale, fraktale und flüssige Matrixstruktur beschreiben. Vertreter der dystopischen Ansicht sprechen von Zeit- und Ortsraffung und bringen die Faktoren Geschwindigkeit und Mobilität ins Spiel.

Der komplexe Charakter dieser Gesellschaft wird hervorgehoben. Das betrifft nicht nur ihre Ontologie und Struktur, sondern auch ihre unzähligen unauflösbaren Widersprüche und Probleme. Daraus ergeben sich die metaphorischen Bezeichnungen der Gesellschaft: „komplexe“, „extrem mobile“, „,virtuelle Gesellschaft“, „Risikogesellschaft“, „Gesellschaft der totalen Kontrolle“, „,Gesellschaft der Simulakren“, „,Gesellschaft der digitalen Ureinwohner" (und Immigranten), „Gesellschaft der Netokraten" und "Gesellschaft des Konsumtariats“. Zu den Schlüsselbegriffen der Raumontologie zählen außerdem die Raummetaphern im Allgemeinen und Raum als Platz des Lebens und der Arbeit: virtueller Raum, Cyberspace, erweiterte Realität, Labyrinth, Web, Matrix, soziale Netzwerke, Datenautobahnen oder -highways, digitale Hubs, digitale Städte und digitale Agora. Bewohnt und benutzt werden diese Räume, Autobahnen und Kreuzungen von digitalen Nomaden.

Im Rahmen der utopischen Ansicht gewinnt der Mensch neue Möglichkeiten zur Selbstverwirklichung und persönlichen Entwicklung. Folglich wird er freier und ausgeglichener. Im Rahmen der dystopischen Ansicht befindet sich der Mensch nicht nur auf dem Weg zur psychischen Instabilität und sozialen Einsamkeit, sondern auch zur digitalen Schizophrenie oder Digiphrenia (Rushkoff 2013).

\subsection{Kompromissposition}

Im vorliegenden Beitrag wird die te chnorationale Kompromiss po sition vertreten. Nach dieser Ansicht ist die Technologisierung ein unvermeidlicher globaler Faktor der Gesellschaftsentwicklung, der sowohl positive als auch negative anthropologische Veränderungen hervorrufen 
kann. Vertreter dieses Standpunkts (M. Castells, A. Nazaretyan, P. Schedrovitsky) sind davon überzeugt, dass ein Gleichgewicht zwischen technischem Fortschritt und geistiger Entwicklung der Gesellschaft möglich ist.

Von diesen drei Ansichten unabhängig sieht die reale Tendenz zu Beginn des 21. Jahrhunderts so aus, dass nur wenige Menschen danach streben, nicht wohnortgebunden zu sein und Telearbeit in einem digitalen Cottage („electronic cottage“) zu verrichten. Diese Weiterentwicklung wurde von vielen Futurologen des 20. Jahrhunderts vorhergesagt. Angestrebt wird die maximale Mobilität und ständige Angeschlossenheit an das globale Netz. Als Arbeitsplatz nutzt man dann dabei viele Orte des globalen hybriden Netzwerks. Mit anderen Worten wird das digitale Nomadentum allmählich zum globalen Trend der Informations- und Kommunikationsgesellschaft.

\section{Die Kultursemiotik als Basis für die Analyse der Kultur von digitalen Nomaden}

\subsection{Gegenstände der Anthropologie und Kultursemiotik}

Die Kultur von digitalen Nomaden kann ohne Zweifel genauso wie alle anderen Kulturen von unterschiedlichen Wissenschaften untersucht werden. Im Fall des digitalen Nomadismus kommen Sozialphilosophie, Sozialpsychologie, Philologie sowie Sozio-, Sozial- und Medieninformatik in Frage. Jede Disziplin legt den Fokus auf den Aspekt dieser Kultur, der ihrem Gegenstandsbereich entspricht. So stellt sich nur eine Frage, und zwar welche Wissenschaft die systematische integrative Beschreibung dieses Phänomens ermöglicht. Basierend auf den Ideen von J. G. Herder (z.B. Herder 1887-1909) und Werken von E. B. Tylor (1871) einerseits und den Schriften von E. Cassirer (z.B. Cassirer 1925 und 1923-1929) und J. Lotman (1970, 1971, 1975 und 2002) andererseits lässt sich behaupten, dass die Anthropologie und die Kultursemiotik in erster Linie über dieses Potenzial verfügen.

Die Anthropologie hat Kultur jedoch lange Zeit nicht als eine ganzheitliche Erscheinung definiert und es bei der Aufzählung von Phänomenen belassen, die diese Kultur ausmachen. Der Gegenstandsbereich der Anthropologie wurde auch nicht eindeutig strukturiert. Erst Ende der 80er, Anfang der 90 er Jahre des 20. Jahrhunderts wurde eine solche Strukturierung von Posner vorgeschlagen (Posner 1990, 1991, 1992, zusammenfassend dargestellt in Posner 2003). Nach Posner umfasst die Anthropologie drei Teildisziplinen: Sozial-, Material- und Kulturanthropologie. Die erste befasst sich mit der sozialen Kultur bzw. mit der G e s ell s ch a ft , die zweite beschäftigt sich mit der materialen Kultur bzw. mit der Zi vili sati o n, während die mentale Kultur bzw. die Mentalität der Gesellschaft im Mittelpunkt der dritten steht. Die Gegenstände der oben genannten Teildisziplinen der Anthropologie lassen sich nicht nur voneinander 
getrennt untersuchen, sondern auch aufeinander beziehen. Es stellt sich heraus, dass eine Kultur im Sinne der Anthropologie ,nichts anderes ist als eine Gesellschaft, die über eine bestimmte Mentalität verfügt und auf deren Basis eine Zivilisation entwickelt hat" (Posner 2003: 48). Noch wichtiger und interessanter erscheint die Tatsache, dass Posner theoretische Zusammenhänge zwischen der auf dieser Weise strukturierten Anthropologie und der Kultursemiotik erfolgreich beweisen konnte. Mit anderen Worten hat er eine Konzeption der semiotischen Beschreibung von anthropologischen Begriffen entwickelt. Sie ermöglicht "die Analyse von Kulturphänomenen“ (Posner 2003: 40). Im Grunde genommen ist die Kultursemiotik eine erweiterte Explikation des Begriffs „Kultur“ mit Hilfe der Zeichentheorie.

\subsection{Die Grundbegriffe und Konzepte der Kultursemiotik}

Die hier folgende Zusammenfassung der kultursemiotischen Grundbegriffe, wie sie von Cassirer, Lotman und Posner verstanden werden, beruht auf den Monografien von Posner Was ist Kultur? Zur semiotischen Explikation anthropologischer Grundbegriffe (Posner 1992) und Kultursemiotik (Posner 2003). Der Gegenstandsbereich der Kultursemiotik sind Zeichenprozesse oder Semiosen im weiten Sinne, sie werden als kulturelle Prozesse verstanden. Jeder Prozess, an dem sich ein Element als Zeichen beteiligt, heißt „Zeichenprozess“. Zu ,jedem Zeichenprozess gehört zumindest ein Zeichen, ein Interpret und eine Botschaft, auf die das Zeichen den Interpreten hinweist" (Posner 2003: 41; die Sperrung steht für den Fettdruck im Original). Wenn der Interpret vom Sender angestrebt wird, übernimmt er die Rolle eines Adressaten. Nicht weniger wichtig sind andere Bestandteile des Zeichenprozesses. Das sind Code, Kanal bzw. Medium, Interpretant und Kontext. Ein Code besteht aus einer Anzahl der Bezeichnenden, einer Anzahl der Bezeichneten und den Regeln, die bestimmen, in welchen Beziehungen sie zueinanderstehen (Posner 2003: 42; Nöth 1990: 206ff.). Ein Kanal bzw. Medium ist eine Konstellation von Faktoren, die über eine große Zahl von Zeichenprozessen hinweg unverändert bleiben (Posner 2003: 43). Eine Interpretation der Botschaft, die mittels Zeichen kommuniziert wird, wird als „Interpretant“ bezeichnet. Es gibt drei Haupttypen der Semiosen: Kommunikation, Signifikation und Indikation. Die Hauptelemente der Kommunikation sind Sender, Adressat, Zeichen, Botschaften, Medium und Kontext. Für die Kommunikation sind sie obligatorisch, die Botschaft muss dabei nicht unbedingt codiert werden. Die Signifikation beruht in erster Linie auf dem Code und kann ohne Sender und Adressat stattfinden. Zeichen, Botschaften, Signifikant und Signifikat, Empfänger, Medium und Kontext dagegen müssen vorhanden sein, damit die Signifikation zustande kommt. Dementsprechend können bei der Indikation, die keinen Code vorsieht, Elemente der Semiose wie Signifikat, Signifikant, Sender und Adressat fehlen. Ohne Zeichen, Botschaften, Empfänger, Medium und Kontext ist sie allerdings nicht möglich (Posner 1992). 
Die Beteiligten an den Zeichenprozessen (d.h. Sender und Empfänger bzw. Adressat) werden „Zeichenbenutzer“ genannt (Posner 2003: 41). Wenn alle einen gemeinsamen konventionellen Code benutzen, gehören sie einer Kultur an. So dient die Verwendung ein und desselben Codes hier als Hauptkriterium für ihre Zuordnung zu einer Kultur (Posner 2003: 42).

Zeichenprozesse, Codes und Medien können als semiotische oder Zeichensysteme agieren. Das „Zeichensystem “ist „[e]ine Menge von Interpreten zusammen mit den von innen interpretierten Zeichen und Botschaften sowie den für die Interpretation relevanten weiteren Umständen" (Posner 2003: 39; siehe auch Prieto 1966: 47f.). Man kann den Begriff „Zeichensystem" in Bezug auf Zeichenprozesse im engeren und im weiteren Sinne anwenden. Im engeren Sinne bedeutet das Zeichensystem die Gesamtheit mehrerer Zeichenprozesse mit demselben Code. Als Beispiel eines Zeichensystems im weiteren Sinne kann ein Code dienen, der selbst als ein Zeichensystem mit seinen eigenen Signifikanten und Signifikaten und den Regeln ihrer Zuordnung fungiert. Medien als Zeichensysteme im weiteren Sinne sind Komplexe von Faktoren, die für einen gewissen Zeitraum über bestimmte Eigenschaften verfügen. Deshalb können sie die internen Zeichenprozesse für diesen Zeitraum bestimmten konstanten Einschränkungen unterordnen (Posner 2003: 46). Zeichensysteme als Gesamtheiten der Zeichenprozesse sind ihrerseits Elemente größerer Strukturen. Diese Strukturen werden "Semiosphären“ oder "semiosische Sphären“ genannt; außerhalb dieser Sphären sind keine semiotischen Prozesse möglich (Posner 2003: 39).

\subsection{Lotmans Theorie des Textes als eine Komponente von Posners Kultursemiotik}

Zur Analyse der Gegenstände der oben genannten anthropologischen Teildisziplinen wendet Posner nicht nur Lotmans Konzeption der Semiosphäre, sondern auch seine breite Theorie des Textes an (Lotman 1970: 64ff.; Lotman 1981: 34ff.). Im Rahmen dieser Theorie kann jedes Artefakt, das über eine Funktion und eine codierte Botschaft verfügt, als ein Text betrachtet werden (Posner 2003: 53). So sieht Posner in einer Gesellschaft eine Gemeinschaft von Zeichenbenutzern, von individuellen und kollektiven Kulturträgern. Die Zivilisation wird nach Posner als eine G e s a $\mathrm{m} \mathrm{t}$ heit von Texten (d.h. von Artefakten) verstanden. Unter Mentalität versteht Posner eine Summe konventioneller Codes oder ein System von Zeichenkonventionen (d.h. Mentefakten), die allen Zeichenbenutzern einer Kultur geläufig sind und von innen benutzt werden. Diese Konventionen kontrollieren das soziale Verhalten von Gesellschaftsmitgliedern und determinieren die Funktionen und Bedeutung von Artefakten. Der semiotische Zusammenhang zwischen den drei Gegenständen besteht darin, dass Zeichenbenutzer von konventionellen Codes abhängig sind, wenn sie Texte verstehen möchten. Folglich kann man jede Kultur als ein 
komplexes Ganzes auffassen. Eine Kultur „besteht aus individuellen und kollektiven Zeichenbenutzern, die Texte produzieren und rezipieren, durch die mit Hilfe konventioneller $\mathrm{Codes} B$ ots chaften mitgeteilt werden, welche den Zeichenbenutzern die Bewältigung ihrer P r o b I e m e ermöglichen“ (Posner 2003: 54; Hervorhebung nachträglich hinzugefügt).

Es ist wichtig zu verstehen, dass jede Kultur ein hochdifferenziertes Zeichensystem ist, das sich selbst in Systeme von Zeichensystemen organisiert und eine flexible Struktur hat. Die Dynamik der Kulturentwicklung entsteht aus der Konkurrenz von Organisationsprinzipien der Zeichensysteme, die eine Kultur bilden und in hierarchischen Beziehungen zueinanderstehen. Bei der Beschreibung dieser Mechanismen nimmt Posner die Konzeption von Lotman als Ausgangspunkt. Nach Lotman lässt sich Kultur auffassen „als eine Hierarchie von Zeichensystemen, als Gesamtheit der Texte und ihrer Funktionen oder als ein bestimmter Mechanismus, der diese Texte hervorbringt“" (Lotman u.a. 1975: 73, zit. nach der deutschen Übersetzung in Posner 2003: 55).

\subsection{Semiotische Mechanismen der Bildung und der Erhaltung kultureller Identität}

Posner versteht Kultur als ein System von Texten und ihren Funkti on en. Diese Ansicht hilft inm bei der Bestimmung der Parameter für die Identität und die Grenzen einer Kultur. Durch die Interpretation der Kultur als eine Hierarchie der Zeichensys te me gelingt es inm, den Charakter der Beziehungen von Kulturen in einer Semiosphäre sowie den Kulturwandel zu erläutern.

Im ersten Fall ist entscheidend, wie man die Kriterien zur Auswahl der Botschaften festlegt, sodass diese Botschaften als Texte gelten und die Identität der Kulturträger ausdrücken. Nach Lotman hängen die Kriterien von den Medien ab, die in einer konkreten Kultur verwendet werden. Texte sind Zeichenkomplexe, die mittels verbreiteter und in einer Kultur hoch angesehener Medien kommuniziert werden. Die Hierarchie der Texte in einer Kultur basiert auf ihren durchschnittlichen Lebenserwartungen, die von Medien gewährleistet werden. Im zweiten Fall wird diese Texthierarchie durch das System von Codes bedingt, das seinerseits eine hierarchische Struktur hat und in semiosischen Sphären organisiert ist. Jede semiosische Sphäre ist von mehreren Schichten der nichtsemiosischen Sphären umgeben. Das macht ein gewisses Weltsegment für die Gesellschaft zugänglich. Die semiotischen Sphären im Zentrum oder an der Peripherie der Kultur sowie die nicht dieser Kultur angehörenden Sphären bestehen aus Zeichensystemen mit Codes, die dieses Segment der Welt mittels ihrer Signifikate strukturieren. Nichtsemiosische außerkulturelle Sphären lassen ihre Weltsegmente unstrukturiert (Lotman u.a. 1975; Lotman 1990; Posner 2003: 58). Kulturelle Veränderungen können wie Veränderungen der Grenzen zwi- 
schen diesen Sphären aufgefasst werden. Leider würde die genauere Beschreibung dieser komplexen Prozesse den Rahmen dieses Artikels sprengen. Daher muss sie auf die folgende Zusammenfassung beschränkt werden.

Jedes Mal, wenn eine Gesellschaft ein für sie neues Weltsegment entdeckt, das vorher dem nicht bekannten a u Berkulturellen Teil der Realität angehörte, verwendet sie den rudimentären Code und verwandelt dieses Segment in die gegenkulturelle Realität. So wird das neue Segment identifiziert und in Verbindung zu anderen bereits bekannten Segmenten gebracht. Folglich geschieht eine allmähliche Erweiterung der Realität. Man entwickelt konventionelle Codes und s e m i o t i s i e r t dadurch die Realität. Der entgegengesetzte Prozess der Entsemiotisierung von Realitätssegmenten wird durch Zerstören solcher Codes verursacht (Posner 2003: 59). Posner bemerkt, dass die Globalisierung den Austausch der wichtigsten kulturellen Codes anregt, was zu einzigartigen hybriden Erscheinungen führt (Posner 2003: 61). Auch innerhalb einer Kultur können Veränderungen stattfinden. Das geschieht wegen der Veränderungen von Codes auf der Achse Zentrum - Peripherie. Die nach dem zentralen Code geschaffenen Artefakte werden detaillierter erarbeitet und dienen als Modell für andere Artefaktstypen (Posner 2003: 61f.). Die Hauptthese zum Thema Kulturmechanismus hat Posner wie folgt formuliert:

[Er bewirkt] eine zunehmende Semiotisierung der Welt [...]. Die Bildung eines Codes (zur Erfassung neuentdeckter Weltsegmente), seine Elaborierung (bei deren Einbeziehung in die kulturellen Sphären), seine Zentralisierung und seine schließliche Ersetzung durch andere Codes (die weniger festgelegt und daher flexibler sind) das ist ein zyklischer Prozeß, der erst dann endet, wenn eine Kultur zu existieren aufhört (Posner 2003: 63f.; die Sperrung steht für den Fettdruck im Original).

Die semiotische Herangehensweise lässt eine Kultur auch als e in e n kollektiven Mechanismus der Informationsspeicherung mit Hilfe von konventionellen Codes explizieren (Posner 2003: 64). In früheren Kulturen hatten ihre Mitglieder selbst die Rolle der Informationsträger. In späteren haben Artefakte (d.h. Texte) diese Rolle übernommen. Wie gut die Information gespeichert wird, hängt von der Anzahl der Repliken und der Frequenz der Textrezeption in Gesprächsakten ab. Außerdem hängt es auch damit zusammen, ob ein Code existiert, der eine erneute Formulierung bei jeder neuen Anwendung verlangt (Posner 2003: 64f.).

Alle Kulturen verwenden Textformulierung, Ritualisierung, Gattungsbildung und andere Verfahren und speichern dadurch bestimmte Handlungsmuster, die sich im Laufe der Evolution als wichtig erwiesen haben. Sie erhalten die Identität der Kultur aufrecht. So bleibt auch die strukturelle Information erhalten, die deren weitere Entwicklung bestimmt. Somit ist die Kultur nicht nur ein kollektiver Mechanismus für die Informationsspeicherung mit Hilfe konventioneller Codes, sondern auch ein „Selektionsapparat" und eine „Überlebensmaschine" der Gesellschaft (Posner 2003: 65). 


\section{Die Kultur von digitalen Nomaden als Zeichensystem: allgemeine Bemerkungen}

Im Kontext der semiotischen Ideen von Cassirer, Lotman und Posner lässt sich die Kultur von digitalen Nomaden wie folgt definieren: als eine hierarchische Gesamtheit der speziellen Zeichensysteme, mit deren Hilfe die Identität von digitalen Nomaden gebildet und aufrecht erhalten wird. Die Haupteigenschaften ihrer Identität sind ständige Mobilität und permanente Erreichbarkeit. Die zweite Funktion der Zeichensysteme besteht darin, dass digitale Nomaden mit deren Hilfe ihre Probleme unter den Voraussetzungen der Informations-, Kommunikations- und Netzwerkgesellschaft lösen können. Im Weiteren wird versucht, die Spezifik dieser Zeichensysteme durch die Beschreibung ihrer Elemente zu erfassen. Außerdem wird an konkreten Beispielen gezeigt, wie einige dieser Funktionen realisiert werden und wie Probleme von digitalen Nomaden durch Zeichensysteme gelöst werden.

\subsection{Digitale Nomaden als Zeichenbenutzer}

Als Zeichenbenutzer bleiben die digitalen Nomaden ständig mobil und an das globale Netz angeschlossen. In der Regel sind sie Mitglieder einer oder manchmal mehrerer virtuellen Communitys, die nach verschiedenen Merkmalen gebildet werden. Folgende Kriterien für ihre Klassifikation können angewandt werden:

- Sozialer Status oder finanzielle Lage (Netokraten und Konsumtariat, Eigentümer oder Manager von Unternehmen ohne Büro, Arbeitssuchende usw.);

- Beruf (Programmierer, Werbetexter, Grafikdesigner, Blogger, verschiedene Experten usw.);

- Gebundenheit an ein Unternehmen (unternehmensgebundene und unabhängige DN);

- Familienstand (DN ohne und mit Familie);

- Sexuelle Orientierung (hetero- und homosexuelle DN);

- $\quad$ Art der schöpferischen Tätigkeit (Maler, Dichter, Musiker usw.);

- Geografisches Wandergebiet von DN („Europäer“, „Asiaten“, „Amerikaner" usw.);

- $\quad$ Art des Nomadenseins (ständige oder saisonale DN);

- Grad des Komforts (gewöhnliche DN oder Flashpacker );

- Typ des mobilen Endgerätes, Vorliebe für bestimmte IT-Marken (Appleoder Samsung-Liebhaber);

- Essgewohnheiten und weitere.

In jeder der oben genannten Communitys als Teil des Zeichensystems haben allgemeine für alle digitalen Nomaden relevante konventionelle Codes 
ihre Gültigkeit. Neben diesen gelten hier zusätzliche konventionelle Codes („Subcodes“ nach Jakobson 1960), die die Spezifik der jeweiligen Gemeinschaft widerspiegeln.

\subsection{Die Codes von digitalen Nomaden}

Man kann davon ausgehen, dass die ursprünglichen Codes des digitalen Nomadismus künstlich und konventionell waren, sich aber mit der Zeit zu alltäglichen Verhaltenscodes entwickelten. Codes werden als Komplexe von Signifikaten, Signifikanten und von den Regeln ihrer Zuordnung zueinander definiert sowie als ein Mittel zum Strukturieren von Botschaften verstanden. Von dieser Auffassung ausgehend können von DN interpretierbare (decodierbare) und angewandte (codierte) abstrakte metaphorische und konkrete Begriffe als kulturelle Schlüs selcodes agieren. Das sind beispielsweise Nomadismus, Nomadenobjekte (drahtlose mobile Endgeräte), Nomadentechnologien (Apps, QR-Codes), Oasen (z.B. Cafés, Bibliotheken, Coworking Spaces, öffentliche Hotspots) und virtuelle Feuerstellen (Mitchell 2003: 158; z.B. Online-Foren, Blogs). Selbst der Ausdruck digitale Nomaden ist ein Diskursmarker, d.h. ein Code, der den Lebensstil von ständig mobilen und an das globale Netz angeschlossenen Menschen beschreibt, der die Grundbedeutungen ihrer Zeichen, Texte und Rituale entschlüsselt und speichert sowie alle diese Bedeutungen in Worte fasst.

Dank der Möglichkeiten, die das Grafikdesign und digitale Technologien eröffnen, sind die visuellen kulturellen Codes von DN im virtuellen Raum der neuen Medien unbegrenzt vielfältig. Es ist anzunehmen, dass genau diese Vielfalt von Codes die Vitalität der Nomadenkultur garantiert. Als Signifikaten treten in diesen Codes Gegenstände und Erscheinungen der realen und virtuellen Welt, aber auch die geistiger und intellektueller Natur auf. Hinzu kommen noch Objekte und Phänomene mit einem hybriden ontologischen Status, das heißt, sie gehören gleichzeitig der realen und der virtuellen Welt an. Ein Beispiel wäre die Technologie der sogenannten „Augmented Reality“ (erweiterte Realität). Alle diese Signifikaten haben zwei Hauptmerkmale gemeinsam, und zwar dass sie für digitale Nomaden einen tieferen Sinn ergeben und ihre Werte wiedergeben. Diese zwei Merkmale machen den Unterschied zwischen den Signifikaten der kulturellen Codes und den Signifikaten der einfachen, nicht an eine Kultur gebundenen Zeichen aus. Die Signifikanten der visuellen Codes sind Abbildungen, die mit 2D- oder 3D-Technologien erstellt worden sind. Diese Abbildungen repräsentieren die reale, virtuelle oder hybride Welt und können dabei sehr abstrakt sein wie z. B. QR-Codes. Die visuellen Codes beschränken sich nicht nur auf das oben Genannte. Beispielsweise können auch das Äußere von digitalen Nomaden (siehe Abbildung 1) oder die visuelle Seite der zwischenmenschlichen Kommunikation, etwa die Gestik, dazu gezählt werden. 


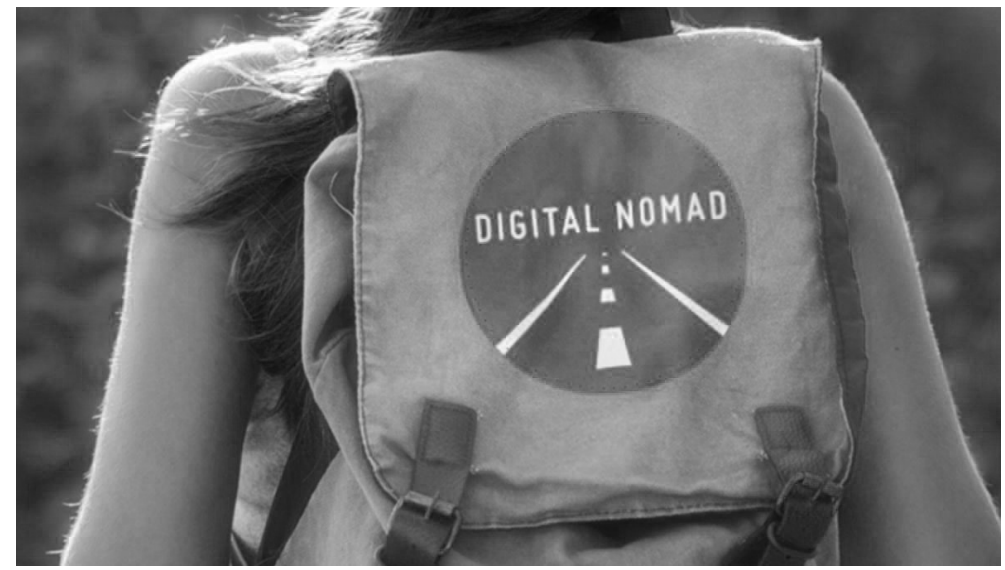

Abb. 1: Die visuellen Codes von digitalen Nomaden.

Jeder Typ der kulturellen Codes von DN ist mit einem bestimmten Typ der Medien verbunden, die durch diese Codes codierte Mitteilungen weitergeben. Einige Codes von DN haben ihren Ursprung in der Synthese der Konventionen von früheren (Sub-)Kulturen. Es handelt sich um Konventionen der digitalen Kultur im Allgemeinen und der Kultur von digitalen Nomaden im Besonderen. Als Beispiel kann man das Zeichensystem der 100 QRCodes nennen. Das wurde 2011 vom US-amerikanischen Medienkünstler Golan Levin ${ }^{2}$ und einigen Freiwilligen speziell für digitale Nomaden entwickelt. Einerseits basiert es auf der Grundlage des visuellen Zeichensystems von Landstreichern und Wanderarbeitern des 19. Jahrhunderts und der Zeit der Großen Depression, auch Hobo-Zeichen genannt. Sie haben besondere Graffiti-Codes (siehe Abb. 2) erfunden, um ihr Leben als Nomaden zu erleichtern.

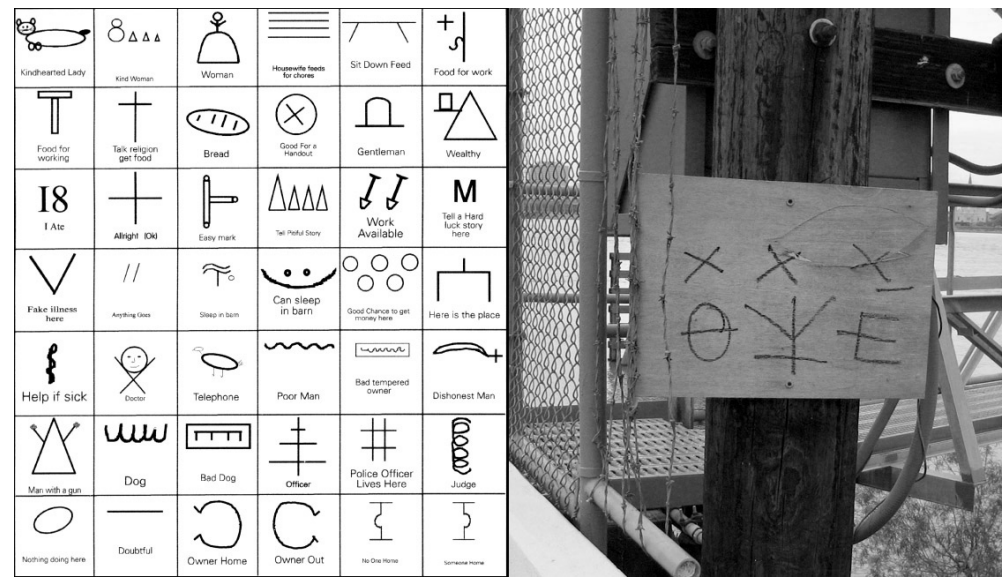

Abb. 2: Hobo-Zeichen. 


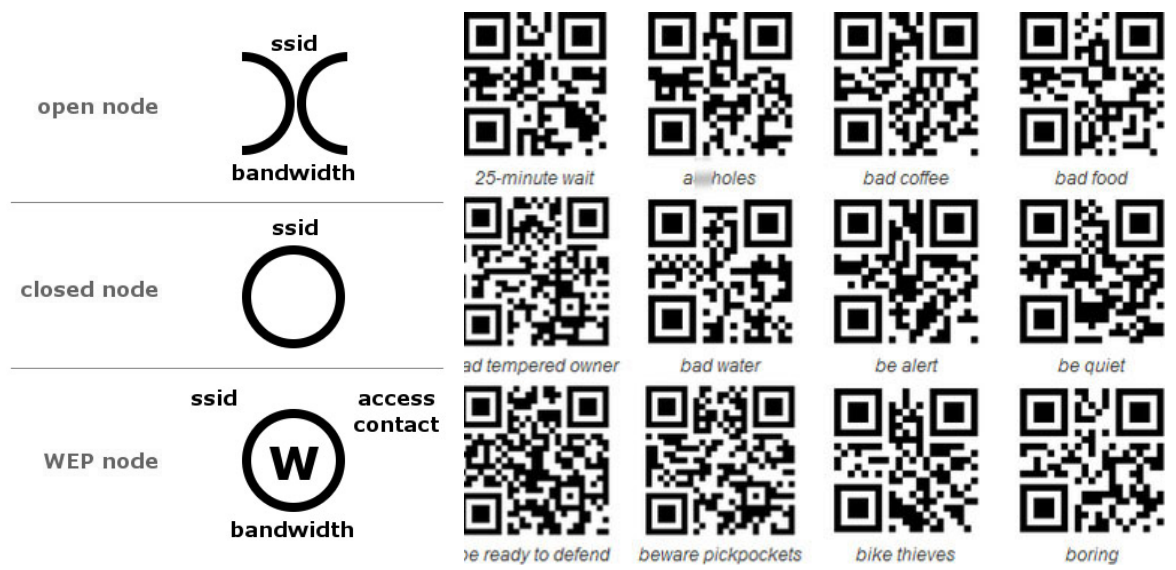

Abb. 3 und 4: WarChalking (links) und Levins QR-Codes.

Andererseits setzt Levins Zeichensystem die Tradition des WarChalking fort. Die Idee des WarChalking schlug der Londoner Informationsarchitekt Matt Jones vor (vgl. Ward 2002). Sie bestand darin, dass an den Wänden, Laternenmasten und auf den Straßen vor Häusern die Hotspotzonen durch mit Kreide gemalte Symbole (Abb. 3) gekennzeichnet werden. Levins QR-Codes sind eine Art zweidimensionaler Barcodes und dienen ihrerseits der Markierung im städtischen Raum, damit digitale Nomaden informiert, auf Richtungen hingewiesen und vor Gefahren gewarnt werden. Bestimmte Apps auf mobilen Endgeräten wandeln diese Codes (Signifikante) in kurze Mitteilungen (Signifikate) um: „Pass auf!“, „Keine Sorge!“, „Schlechtes Essen“, „Guter Kaffee“, „Kostenloses WLAN“, ,Versteckte Videoüberwachung“, ,Toilette in der Nähe“, „Veganer: Vorsicht!" und Ähnliches (Abbildungen 4 und 5). Dieses Beispiel veranschaulicht, wie mit Hilfe von bestimmten kulturellen Codes einige Probleme von digitalen Nomaden gelöst werden können.

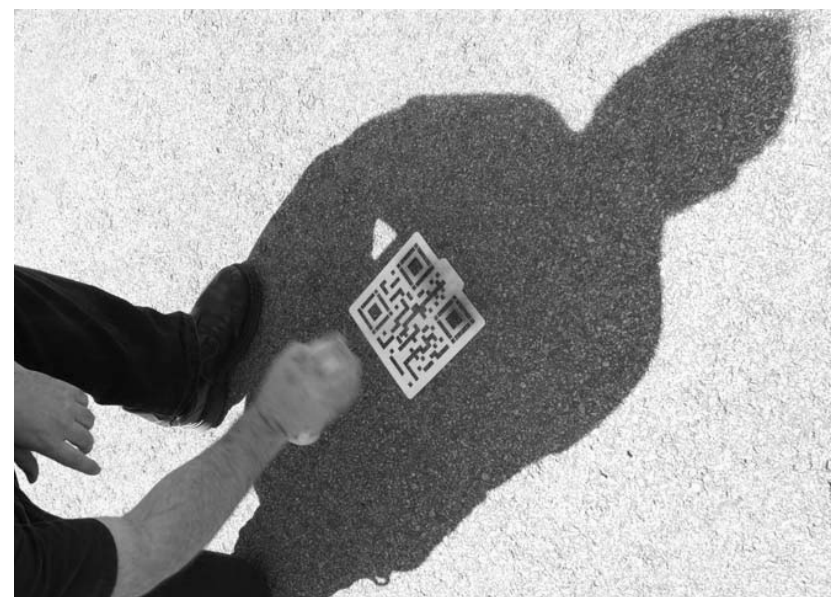

Abb. 5: Levins QR-Codes. 
Außerdem ist das ein Beweis, dass die Kultur von DN trotz ihrer Neuheit gewisse Traditionen aufweist, die manchen älteren Kulturen innewohnen.

\subsection{Die Medien von digitalen Nomaden}

Von allen Medien (bzw. Kanälen), mit deren Hilfe Zeichenprozesse von digitalen Nomaden erfolgen, spielen die technologischen Medien eine besondere Rolle. Zu den t e c h n o l o g i s c h e n Medien zählen Computer, Laptops, mobile Endgeräte und verschiedene andere elektronische Geräte und Gadgets. Ihre Besonderheit ist, dass sie für digitale Nomaden nicht nur eine technische und semiotische, sondern auch eine phatische Funktion erfüllen. Wie ausgeprägt die Letztere ist, äußert sich vor allem dann, wenn das Gerät plötzlich verloren geht. Das kann bei digitalen Nomaden ein Gefühl erwecken, als ob ihr Zuhause verloren gegangen sei. Technologische Medien sind manchmal schwer von physikalischen zu unterscheiden. Zu physikalischen Medien kann bedingt der drahtlose Internetzugang WLAN gezählt werden, der eine Art Kapital der Netzwerkgesellschaft ist. Die wichtigsten f u n kt i o n a l e n Medien sind Blogs und Foren. Sie charakterisieren die Zeichenprozesse je nach Ziel und Form der Mitteilungen. Beispiele von kodifizierten Medien sind private oder Firmenblogs von digitalen Nomaden, ihre lokalen stadtbezogenen oder globalen Internetforen, Blogs von Experten für die Entwicklung von Nomadenrouten in einer Stadt oder Blogs von Alpinisten-Flashpackern usw. Was digitale Nomaden selbst als b i o lo g i s che Medien betrifft, sind bei innen visuelle, auditive und taktile Rezeptoren am besten entwickelt. Als grundlegende s o z i a le Medien fungieren dritte Orte, und zwar Cafés mit Internetzugang wie Starbucks, Coworking Spaces, Geschäfte, in denen man Nomadenobjekte kaufen kann. Berücksichtigt man die duale Ontologie der Netzwerkgesellschaft (virtuelle Realität und reale Virtualität), muss man PCund Handybildschirme zu sozialen Medien zählen. Sie übernehmen die Rolle des Interfaces, d.h. der Schnittstelle zwischen der realen und der virtuellen Welt, in denen semiotische Prozesse zugleich auch als Prozesse der sozialen Kommunikation ablaufen können. Die sozialen Medien müssen besonders hervorgehoben werden, weil sie alle anderen Medientypen organisieren, die an der Zeichenproduktion beteiligt sind (vgl. Posner 2003: 45).

Das Supermedium für digitale Nomaden ist das Internet als ein komplexes Zeichensystem, das über Eigenschaften verfügt, die das Ablaufen von semiotischen Prozessen determinieren. Dieses Supermedium ermöglicht momentane virtuelle Kontakte zur Organisation der semiotischen Prozesse. Dabei kann eine unbegrenzte Anzahl von digitalen Nomaden als Zeichenbenutzer involviert sein, unabhängig davon, in welchem Teil der Erde sie sich befinden. Demnach ist das Internet auch als eine Semiosphäre zu betrachten, also als eine Hierarchie verschiedener Zeichensysteme, die von digitalen Nomaden unterschiedlicher Subkulturen anhand von Subcodes generiert werden. 


\subsection{Artefakte und Mentefakte von digitalen Nomaden}

Im Rahmen Lotmans breiter Theorie des Textes, auf die auch Posner Bezug nimmt, können alle von und für digitale Nomaden geschaffenen Artefakte als Texte betrachtet werden. Die Summe aller Artefakte und Technologien einer bestimmten Gesellschaft macht bekanntlich ihre Zivilisation aus (Posner 2003: 50). Die hybride Ontologie der Netzwerkgesellschaft (vgl. Punkt 2.2 dieses Artikels) determiniert den hybriden Status der Zivilisation von digitalen Nomaden. Sie existiert demnach in der re a l e $n$ und virtue II en Welt. Infolgedessen weist sie nicht nur materielle Artefakte wie z. B. verschiedene Geräte, Gadgets und andere Nomadengegenstände, sondern auch virtuelle Artefakte auf. Sie entstehen durch digitale Technologien, es sind beispielsweise Audio- und Video-Dateien, digitale Texte, Computergrafiken usw. Die Anzahl der virtuellen Artefakte übersteigt schon lange die der materiellen und analogen Artefakte. Aus diesem Grund kann die moderne Epoche analog zur Stein- oder Bronzezeit „Digitalzeit“ genannt werden.

So wie die Zivilisation aus Artefakten und Technologien besteht, machen M e n t e f a k te und Konventionen die Mentalität der Gesellschaft aus (vgl. Posner 2003: 49). Die Mentefakte der Kultur von DN sind Ideen und Werte, die ihr alltägliches mobiles Nomadenleben bestimmen. Unter Ideen versteht Posner alle Kategorien, die eine Gesellschaft nutzt, um sich selbst und ihre eigene Realität zu interpretieren. Bei dieser Betrachtungsweise zählen nicht nur die Idee der Ortsunabhängigkeit, sondern auch solche Kategorien wie Nomadismus, Nomadenobjekte, mobiles Haus, Oasen und virtuelle Feuerstellen dazu. Sogar die Begriffe Internet, WLAN und Ladegerät haben für DN den Status lebenswichtiger Werte (Abb. 6 und 7). Diese Begriffe können zugleich als zentrale Codes für die Kultur der Digitalen Nomaden angesehen werden: Jede Mentalität lässt sich als Menge von Codes auffassen (vgl. Posner 2003: 54).

Artefakte von digitalen Nomaden sind leicht in andere Zivilisationen und Kulturen übernehmbar, das gilt selbst für sehr langlebige und prägende
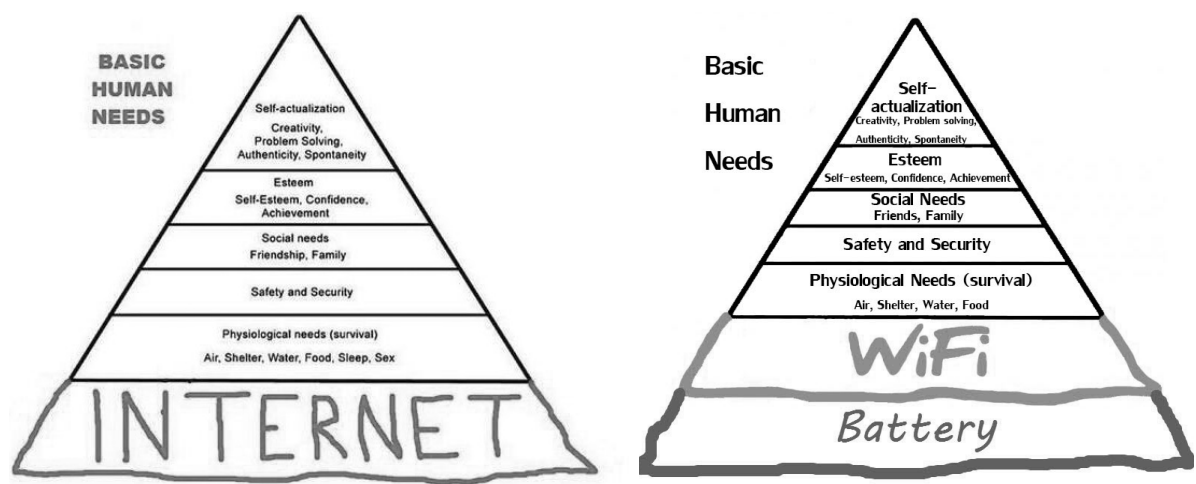

Abb. 6 und 7: Lebenswichtige Werte für digitale Nomaden. 
Kulturen. Muslimische Pilger benutzen auf ihren Handys oft den Qibla-Kompass zur Bestimmung der Gebetsrichtung auf die Kaaba in Mekka. Für Katholiken gibt es eine App, um SMS-Mitteilungen vom Papst zu erhalten. Dabei ergänzen Artefakte und Mentefakte unterschiedlich stark etablierter Kulturen einander. Wenn es allerdings um weniger beständige Kulturen wie Teenager- oder Unternehmenskulturen geht, können hier unter dem Einfluss der Nomadenkultur fundamentale anthropologische Veränderungen auftreten. Diese stellen viele Autoren fest (vgl. Braidotti 1994; Rosseel 2000; Rushkoff 2013 u.a.)

\subsection{Grenzen und Mechanismen der Globalisierung der Kultur von digita- len Nomaden}

Die Identität der Kultur von digitalen Nomaden wird in erster Linie durch Nomadenobjekte (z.B. mobile Endgeräte, Laptops, Modems, Ladegeräte, USB-Sticks) als wichtige Artefakte gewährleistet. Aus dieser Perspektive betrachtet erfolgt in der Nomadenkultur die Se lbsterze ugung von Texte $n$, das heißt, Texte bringen andere Texte hervor. Die Grenzen der oben genannten Identität fallen mit den Grenzen zwischen Texten und NichtTexten dieser Kultur zusammen. Sie werden z. B. durch folgende Oppositionen bestimmt: „mobil“ versus „immobil“, „angeschlossen“ versus „,nicht angeschlossen“, „online“ versus „offline“, „drahtlos“ versus „drahtgebunden“, „digital“ versus „analog“, ,polyfunktional“ versus „monofunktional“, „kompakt“ versus „,voluminös“, „schnell“ versus „langsam“, „frei“ versus „gebunden“ usw. Linke Glieder der angeführten Oppositionen sind Kategorien, die Merkmale der Artefakte-Texte in der Kultur von DN beschreiben. Rechte Glieder der Oppositionen definieren Merkmale der Nicht-Texte.

Wie bereits im Artikel diskutiert, spielen moderne mobile Endgeräte und Technologien für digitale Nomaden nicht nur die Rolle der Artefakte und Medien bzw. Kanäle, sondern auch die der kulturellen Codes. Mobile Geräte und Technologien verfügen über breite Möglichkeiten zum Replizieren und schnellen Empfangen von Texten und somit auch zum Speichern von Informationen mit Hilfe von konventionellen Codes. In ihrer Gesamtheit stellen sie einen kollektiven Gedächtnismechanismus dar, der die Identität der Kultur von DN bewahrt. Auf der anderen Seite bilden sie auch die oben erwähnte Kriegsmaschine, die in der Lage ist, jeden anderen kulturellen Code zu zerstören (vgl. Deleuze und Guattari 1986 und 1992). Da mobile Endgeräte und Technologien die Funktionen von Artefakten-Texten, Medien und von kulturellen Codes in sich vereinen, sind sie deshalb gleichzeitig ein Mechanismus der Globalisierung der gegebenen Kultur. Vom Standpunkt der Kultursemiotik aus muss diese Globalisierung als fortschreitende Semiotisierung von neuen Segmenten der hybriden Ontologie der Netzwerkgesellschaft verstanden werden. 


\section{Abschlussbemerkung}

Die Überlegungen in diesem Beitrag weisen darauf hin, dass die Methoden der kultursemiotischen Analyse im Sinne von Lotman und Posner und die ontologische Herangehensweise die Beschreibung der spezifischen Kultur von digitalen Nomaden in ihrer Gesamtheit ermöglichen. Mit diesen Methoden lassen sich darüber hinaus auch die Mechanismen der Globalisierung dieser Kultur erklären. Der Artikel beinhaltet nur allgemeine Bemerkungen zum semiotischen Modell der Kultur von digitalen Nomaden im Kontext der Ontologie der Netzwerkgesellschaft. Eine ausführlichere Auseinandersetzung mit dem Thema hätte den Rahmen des Artikels gesprengt, was mit der Größe und Komplexität des Untersuchungsgegenstandes zusammenhängt. Der Artikel zeigt allerdings die Perspektiven für die weiterführende Forschung in diesem Bereich auf.

Aus dem Russischen übersetzt von Alexandra Haunstein

\section{Anmerkungen}

1 Als „Flashpacker“ werden Individualreisende bezeichnet, die weniger Zeit, aber mehr Geld zur Verfügung haben als klassische Backpacker (Rucksacktouristen), und meist etwas älter sind (jenseits der 30).

2 Dazu Mitchell: „In traditional nomadic societies, regularly rekindled campfires provided mobile focal points for social life. [...] In the mobile wireless era, a third alternative has emerged; we can use our portable communication devices to construct meeting points and gathering places on fly - places that may only be known within particular, electronically linked groups“ (Mitchell 2003: 158).

3 Siehe unter: http://aksioma.org/fuck/; http://www.flong.com/projects/qr-codes-fordigital-nomads/.

\section{Literatur}

Attali, Jacques (1991), Millennium: winners and losers in the coming world order. New York: Random House.

Bard, Alexander und Jan Soderqvist (2002), Netocracy: the new power elite and life after capitalism. London: Pearson FT Press.

Baron, Naomi S. (2001), Alphabet to Email: How Written English Evolved and Where It's Heading. London u. a.: Routledge.

Baudrillard, Jean (1981), For a Critique of the Political Economy of the Sign. St Louis: Telos.

Bauman, Zygmunt (1993), Postmodern Ethics. London: Routledge.

Bauman, Zygmunt (2000), Liquid Modernity. Cambridge: Polity Press. Deutsch von R. Kreissl: Flüchtige Moderne. Berlin: Suhrkamp 2005. 
Bell, Daniel (1973), The Coming of Post-Industrial Society: A Venture in Social Forecasting. New York: Basic Books.

Braidotti, Rosi (1994), Nomadic Subjects: Embodiment and Sexual Difference in Contemporary Feminist Theory. New York: Columbia University Press.

Brzezinski, Zbigniew (1970), Between Two Ages: America's Role in the Technetronic Era. New York: Viking Press.

Cassirer, Ernst (1925), Sprache und Mythos: Ein Beitrag zum Problem der Götternamen. Leipzig und Berlin: Teubner.

Cassirer, Ernst (1923-1929), Philosophie der symbolischen Formen. Berlin: Bruno.

Castells, Manuell (1996-1998), The Information Age: Economy, Society and Culture. 3 Bde. Malden, MA u.a.: Blackwell.

Celento, David (2008), Urban Dwellings for the Digital Nomad. Wood Structure Symposium 2008: Without a Hitch: New Directions in Prefabricated Architecture: <http:// scholarworks.umass.edu/wood/2008/sept27/6/>

De Lange, Michiel (2009), „'Digital Nomadism': a Critique“. Draft version of a section of dissertation. URL: <http://www.bijt.org/wordpress/writing/>

Deleuze, Gilles und Felix Guattari (1986), Nomadology: The War Machine. New York: Semiotext(e).

Deleuze, Gilles und Felix Guattari (2004), Capitalism and Schizophrenia. A Thousand Plateaus. London u.a.: Continuum. Deutsch von G. Ricke und R. Vouillé: Tausend Plateaus: Kapitalismus und Schizophrenie. 6. Auflage. Berlin: Merve 2005.

Fortunati, Leopoldina (2002), „The Mobile Phone: Towards New Categories and Social Relations“. Information, Communication \& Society 5, 4: 513-528.

Galbraith, John Kenneth (2007), The New Industrial State. Princeton: Princeton University Press.

Garfinkel, Simson (2000), Database Nation: The Death of Privacy in the 21st Century. Beijing: O’Reilly \& Associates.

Herder, Johann Gottfried (1887-1909), „Ideen zur Philosophie der Geschichte der Menschheit“. In: Suphan, Bernhard (Hrsg.), Herder. Sämtliche Werke. Berlin: Weidmann.

Huxley, Aldous (2005), Brave New World and Brave New World Revisited. New York: Harper Perennial Modern Classics.

Johannessen, Steinar (2000). The Nomadisation of Worklife: Advantages and disadvantages of mobile telework. University of Oslo/University of East London. Dissertation.

Jakobson, Roman (1960), „Linguistics and Poetics“. In: Thomas A. Sebeok (ed.), Style in Language. Cambridge: MIT Press: 350-377. Deutsch von T. Schelbert: „Linguistik und Poetik“. In: Roman Jakobson: Poetik: ausgewäh/te Aufsätze 1921-1971. 2. Auflage. Frankfurt am Main: Suhrkamp 1990: 83-121.

Jaspers, Karl (1953), The Origin and Goal of History. New Haven, CT:Yale University Press. Kahn, Herman und Barry Bruce-Briggs (1972), Things to Come. Thinking about the Seventies and Eighties. New York: Macmillan.

Kakihara, Masao und Carsten Sorensen (2001), „Expanding the 'mobility' concept“. ACM SIGGROUP Bulletin Archive 22, 3: 33-37.

Kuzheleva-Sagan, Irina P. (2014), „Network Society. Evolution of Views: Concepts, Images, Metaphors". Cloud-Cuckoo-Land, International Journal of Architectural Theory 19, 32: $25-41$. 
Lotman, Jurij M. (1970), Stat'i po tipologii kul'tury. Materialy k kursu teorii literatury. Bd. 1. Tartu: Tartuskii Gos. Universitet.

Lotman, Jurij M. und Boris A. Uspenskij (1971). „O semiotičeskom mechanizme kul'tury“. Trudy po znakovym sistemam 5: 144-166.

Lotman, Jurij M. u.a. (1975), „Theses on the Semiotic Study of Cultures (as Appliedto Slavic Texts)“. In: Sebeok, Thomas. (ed.), The Tell-Tale Sign. A Survey of Semiotics. Lisse: Peter de Ridder: 57-83.

Lotman, Jurij. M. (2002), Stat'i po semiotike kul'tury i iskusstva. St. Petersburg: Academic Project.

Makimoto, Tsugio und David Manners (1997), Digital Nomad. New York: Wiley.

Marcuse, Herbert (1991), One-dimensional Man: Studies in the Ideology of Advanced Industrial Society. London: Routledge.

McLuhan, Marshall (1994), Understanding Media: The Extensions of Man. Cambridge, Mass.: MIT Press.

Meyrowitz, Joshua (1985), No Sense of Place: The Impact of Electronic Media on Social Behavior. New York: Oxford University Press.

Mitchell, William J. (2003), Me++: The Cyborg Self and the Networked City. Cambridge u.a.: MIT Press.

Mohn, Tanya (2014), „How to Succeed at Becoming a Digital Nomad“. Forbes/Lifestyle. URL: <http://www.forbes.com/sites/tanyamohn/2014/03/19/tips-for-becoming-asuccessful-digital-nomad/\#c7e44ad27443>

Naisbitt, John (1982), Megatrends. Ten New Directions Transforming Our Lives. New York: Warner.

Nazaretyan, Akop P. (2004), „Antropogennye krizisy: gipoteza techno-gumanitarnogo balansa“. Vestnik Rossijskoj Akademii Nauk 74, 4: 319-330.

„Nomads at last. Special report“. The Economist(10 April 2008). URL: <http://www.economist.com/node/10950394>

Orwell, George (2003), Nineteen eighty-four. New York: Plume.

Peters, John D. (1999), „Exile, Nomadism, and Diaspora: the Stakes of Mobility in the Western Canon“. In: Hamid Naficy (ed.), Home, Exile, Homeland: Film, Media, and the Politics of Place. New York: Routledge: 17-41.

Posner, Roland (1991), „Kultur als Zeichensystem. Zur semiotischen Explikation kulturwissenschaftlicher Grundbegriffe". In: Aleida Assmann und Dietrich Harth (Hrsg.), Kultur als Lebenswelt und Monument. Frankfurt a.M.: Fischer: 37-74.

Posner, Roland (1992), „Was ist Kultur? Zur semiotischen Explikation anthropologischer Grundbegriffe“. In: Marlene Landsch u.a. (Hrsg.), Kultur-Evolution. Fallstudien und Synthese. Frankfurt a. M.: Lang: 1-65.

Posner. Roland (2003), „Kultursemiotik“. In: Ansgar and Vera Nünning (eds.), Konzepte der Kulturwissenschaften: Theoretische Grundlagen - Ansätze - Perspektiven. Stuttgart und Weimar: Metzler: 39-71.

Prieto, Luis J. (1966), Messages et signaux. Paris: Presses Universitaires de France.

Rosseel, Eric (2000), „Nomadisation: Social, Psychological and Cultural Context for the XXI Century?" In: Ljubava Moreva (ed.), Symbols, Images and Stereotypes: Historical and Existential Experience. International Readings on Theory, History and Philosophy of Culture 8. St. Petersburg:12-28. 
Rushkoff, Douglas (2013), Present shock: When everything happens now. New York: Current.

Sassen, Saskia (2001), The Global City: New York, London, Tokyo. Princeton: Princeton University Press.

Schedrovicky, Pëtr G. (2000), „Gosudarstvo v èpochu gumanitarnych technologij“. Russkij žurnal(21.07.2000). URL: <http://old.russ.ru/politics/meta/20000721_sch.html> Toffler, Alvin (1980), The Third Wave. New York: Bantam Books.

Tylor, Edward B. (1871), Primitive Culture. London: Murray.

Urry, John (2000), Sociology beyond Societies. Mobilities for the Twenty-first Century. London u.a.: Routledge.

Virilio, Paul (2010), The Futurism of the Instant: Stop-Eject. Cambridge: Polity.

Waller, Mark (1998), „Corporate nomads with the skill to step into the breach“. The Times 31 (13.10.1998).

Ward, Mark (2002), Write here, right now. BBC News (01.07.2002): <http://news.bbc. co.uk/2/hi/in_depth/sci_tech/2000/dot_life/2070176.stm>

Wood, Martin (2005), „Nomad Aesthetics and the Global Knowledge Economy“. TAMARA: Journal of Critical Postmodern Organization Science 3, 4: 50-64.

\title{
Bildquellen
}

Abb. 1: <https://www.linkedin.com/pulse/20140204142733-758147-digital-nomads-awork-freedom-revolution-video>

Abb. 2: <http://infoczarina.blogspot.ru/2008/03/term-hobo-has-unknown-origin-perhaps. $\mathrm{html}>$

Abb. 3: <http://www.goblinbyte.com/2015/02/warchalking-hobo-symbols-of-happiness. $\mathrm{html}>$

Abb. 4: <http://weburbanist.com/2012/04/16/qr-hobo-codes-secret-symbol-stencils-fordigital-nomads/>

Abb. 5: <http://www.flong.com/projects/qr-codes-for-digital-nomads/>

Abb. 6: <https://twitter.com/ciarfitz/status/459431452086919168>

Abb. 7: <http://www.houstonpr.co.uk/updated-maslows-hierarchy-of-needs-extended/>

\author{
Dr. Irina P. Kuzheleva-Sagan \\ Staatliche Universität Tomsk \\ Lehrstuhl für soziale Kommunikation \\ Ul. Lenina 36 \\ 634050 Tomsk, Russland \\ E-Mail: ipsagan@mail.ru
}

